\title{
Impacto de la fermentación y secado sobre el contenido de polifenoles y capacidad antioxidante del clon de cacao CCN-51
}

\section{Impact of fermentation and drying in polyphenol content and antioxidant capacity of cocoa variety $\mathrm{CCN}-51$}

\section{Impacto da fermentação e secagem no teor de polifenóis e capacidade antioxidante do clone de cacau $\mathrm{CCN}-51$}

\author{
Andrea Pallares Pallares ${ }^{1}$; Mauren R. Estupiñán $A^{1}$; Janeth Aidé Perea Villamil2; \\ Luis Javier López Giraldo ${ }^{\text {* }}$

\begin{abstract}
${ }^{1}$ Escuela de Ingeniería Química. ${ }^{2}$ Escuela de Química. Universidad Industrial de Santander. Grupo en Ciencia y Tecnología de Alimentos-CICTA. Ciudad Universitaria, Calle 9 Carrera 27. Bucaramanga, Colombia.
\end{abstract}

*ljlopez@uis.edu.co

Fecha Recepción: 18 de febrero de 2016

Fecha Aceptación: 27 de octubre de 2016

\begin{abstract}
Resumen
La influencia del tiempo de fermentación y secado fue evaluada simultáneamente sobre la capacidad antioxidante $(C A)$ y el contenido de polifenoles totales $(P T)$ de cacao clon $\mathrm{CCN}-51$ (origen ecuatoriano resultante del cruce ICS95xIMC67), buscando seleccionar condiciones de beneficio que permitan obtener un producto de mayor valor agregado desde el punto de vista funcional. Los polifenoles presentes fueron cuantificados usando el método de Folin-Ciocalteu $(P T)$ y tentativamente identificados por LC-MS, mientras que la capacidad antioxidante fue analizada usando el método ORAC. El beneficio comprendió las etapas de microfermentación en cajón y secado natural al sol. Los análisis siguieron un diseño experimental factorial multinivel de 15 experimentos/muestreo. Las diferencias significativas entre niveles fueron establecidas con un análisis de varianza. La CA (expresados en micromoles equivalentes de trolox (TE)/gramos de muestra seca) y el contenido de $P T$ (expresados en miligramos equivalentes de ácido gálico (EAG)/gramo de muestra seca) variaron significativamente durante la fermentación,

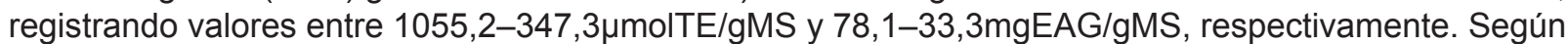
el ANOVA, el efecto del secado natural fue poco significativo en la evolución de dichas variables $(p<0,05)$. La evolución del contenido de PT y la $C A$, como una función de los días de fermentación, se ajustó a un modelo matemático recíproco $\left(R^{2}>0,95\right.$ en ambos casos). Las ecuaciones ajustadas fueron usadas para predecir la evolución del contenido de $P T$ de clones tipo Forastero y Amazónico, cuando se comparó con resultados experimentales de otros trabajos, el error relativo porcentual promedio fue de $20 \%$. Finalmente, se estableció que la fermentación ocasiona una disminución en los monómeros y oligómeros presentes (excepto catequina y procianidina B1) que puede relacionarse con la disminución de la CA. La fermentación es la etapa del beneficio que mayor impacto genera en la variación del contenido de $P T$ y la $C A$.
\end{abstract}

Palabras clave: cacao, beneficio, CCN-51, polifenoles totales, capacidad antioxidante.

\begin{abstract}
The influence of fermentation and drying time was evaluated simultaneously on the antioxidant capacity $(C A)$ and total polyphenol content $(P T)$ clone $C C N-51$, seeking conditions to benefit leading to a higher valueadded product from the point functionally. ORAC $(C A)$ and Folin-Ciocalteu $(P T)$ methods were employed.
\end{abstract}


Polyphenols were tentatively identified by LC-MS. The profit was realized in stages microfermentation drawer and natural drying in the sun. The analysis followed a multi-factorial experimental design of 15 experiments/sampling. Significant differences between levels were established with an analysis of variance. The $C A$ (expressed in micromole equivalents of trolox (TE)/grams of dried sample) and $P T$ content (expressed in gallic acid equivalent (GAE)/grams of dried sample) varied significantly during

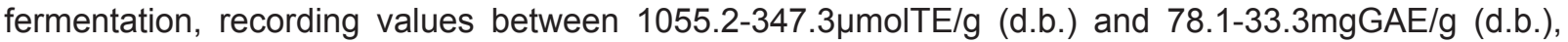
respectively. According to ANOVA, the natural drying effect was not significant in the evolution of these variables $(\mathrm{p}<0.05)$. The evolution of the content of $P T$ and $C A$, as a function of days of fermentation, a reciprocal mathematical model was adjusted $\left(R^{2}>0.95\right.$ in both cases). The fit equations were used to predict of behavior of $P T$ in Forastero and Amazonico clones, when results were compared with experimental results of other works an average relative error of $20 \%$, was obtained. Finally, it was established that the fermentation causes a decrease in the monomers and oligomers present (except catechin and procyanidin B1) that may be related to decrease $C A$. Fermentation is the stage of profit generated greater impact on the variation of the content of $P T$ and $C A$.

Keywords: cocoa, post-harvest process, CCN-51, total polyphenols, antioxidant capacity.

\section{Resumo}

A influência do tempo de fermentação e secagem foi avaliado simultaneamente sobre a capacidade antioxidante $(C A)$ e o conteúdo total de polifenóis $(P T)$ clone $C C N-51$, que pretende seleccionar condições beneficio que conduzem a um produto de maior valor acrescentado a partir do ponto funcionalmente. ORAC $(C A)$ e Folin-Ciocalteu $(P T)$ métodos foram empregados. Os polifenóis foram tentativamente identificados por LC-MS. O lucro realizado em etapas microfermentación gaveta e secagem natural ao sol. A análise seguiu um delineamento experimental multi-fatorial de 15 experimentos/amostragem. Diferenças significativas entre os níveis foram estabelecidos com uma análise de variância. AC (expressos em micromoles de Trolox/gMS de amostra seca) e conteúdo PT (expresso em miligramas equivalentes de gálico / grama de ácido amostra seca) variaram significativamente durante a fermentação, registrando valores entre 1055,2-347,3 $\mu \mathrm{molTE} / \mathrm{gMS}$ e 78,1-33,3mgEAG/gMS, respectivamente. De acordo com a ANOVA, o efeito de secagem natural não foi significativa na evolução destas variáveis $(p<0,05)$. A evolução do conteúdo de $P T$ e de $C A$, como uma função dos dias de fermentação, foi ajustado um modelo matemático recíproco ( $R^{2}>0,95 \mathrm{em}$ ambos os casos). As equações ajustadas foram utilizados para prever a evolução do teor de PT e os clones de tipo Amazon Forasteiros, quando comparados com os resultados de outros estudos experimentais, a percentagem média de erro relativo foi de $20 \%$. Finalmente, foi estabelecido que a fermentação provoca uma diminuição nos monômeros e oligômeros presentes (exceto catequina e procyanidin B1) que podem estar relacionados à diminuição da $C A$. $\mathrm{A}$ fermentação é o estágio de lucro maior impacto gerado sobre a variação do teor de $P T$ e $C A$.

Palabras-chave: cacau, lucro, CCN-51, polifenóis totais, capacidade antioxidante.

\section{Introducción}

En las últimas tres décadas los trabajos de investigación relacionados con la determinación de las propiedades funcionales del cacao se han incrementado de forma apreciable [1-4]. Esto, porque se ha reconocido que dicho material vegetal puede considerarse como un alimento funcional gracias a que es una fuente natural de antioxidantes, específicamente polifenoles $[5,6]$. Los granos de cacao y los productos elaborados a partir de éstos contienen cantidades importantes de flavanoles, cuyo espectro se extiende desde simples monómeros hasta formas oligoméricas y poliméricas llamadas procianidinas. Entre los monómeros sobresalen cuantitativamente los flavan-3-oles epicatequina y catequina; mientras las procianidinas B1 y B2 son los oligómeros más abundantes [3].

Las propiedades antioxidantes del cacao son afectadas por factores como el genotipo, las condiciones agroclimáticas, el proceso de beneficio (fermentación y secado) y el proceso de industrialización [1,7]. Durante el beneficio y la subsecuente etapa de tostado se registra una pérdida sustancial de los componentes polifenólicos presentes en el grano, disminuyendo por ende la capacidad antioxidante del mismo $[1,8,9]$. 
Entre los 22 clones de cacao recomendados por la Federación Nacional de Cacaoteros de Colombia para ser sembrados en las 4 zonas agroecológicas del país, se encuentra el CCN-51.

Esta variedad es preferida por un gran número de cultivadores debido a su alto rendimiento y tolerancia a las enfermedades [10]. Sin embargo, su utilización como materia prima en la elaboración de chocolatería fina ha recibido críticas de parte de algunos sectores, dadas sus pobres características de sabor y aroma (bajas tonalidades de sabor a fruta y rasgos florales, sumadas a un excesivo amargor y alta astringencia). Por tal razón, entender la evolución del contenido de polifenoles totales $(P T)$ y la capacidad antioxidante $(C A)$ del clon de cacao $\mathrm{CCN}-51$ durante las etapas de fermentación y de secado, es el punto de partida para la elaboración de productos enriquecidos con polifenoles usando este clon y se constituye en el propósito de este trabajo. Se espera de este modo darle mayor valor agregado a un material que está siendo ampliamente cultivado en el país. A la fecha no se ha realizado ningún trabajo de investigación con este objetivo.

\section{Materiales y métodos}

\section{Reactivos, solventes y estándares}

La solución buffer de fosfato $(\mathrm{pH} 7,2)$, Trolox®, fluoresceína sódica y 2,2'-azobis (2-amidinopropano) dihidrocloruro (AAPH) se adquirieron de Sigma-Aldrich. El reactivo de FolinCiocalteu, carbonato de sodio, etanol, n-hexano, ácido gálico, ácido fórmico y acetonitrilo fueron comprados a Merck (Bogotá, Colombia). Todos los reactivos fueron de grado analítico.

\section{Beneficio de los granos de cacao}

La recolección y tratamiento de las muestras del clon de cacao CCN-51 (Origen ecuatoriano resultante del cruce ICS95xIMC67) fueron realizadas en la granja experimental Villa Mónica de la Federación Nacional de Cacaoteros de Colombia ubicada en San Vicente de Chucurí, Santander (693msnm, $\mathrm{T}_{\text {media }} \approx 27^{\circ} \mathrm{C}$ ). Se hicieron dos muestreos en la época de cosecha comprendida entre octubre de 2012 y enero de 2013.

Se empleó el método de microfermentación en cajones propuesto por el Departamento de Investigación de FEDECACAO [11]. El tiempo de desgrane no superó las $24 \mathrm{~h}$ y los granos de
CCN-51 obtenidos $(2,5 \mathrm{~kg})$ fueron ubicados en mallas plásticas de $25 \times 50 \mathrm{~cm}$. Inicialmente, las muestras se dejaron escurrir por 16-18h. Luego fueron presecadas al sol por $30 \mathrm{~h}$ (temperatura promedio del tratamiento $27^{\circ} \mathrm{C}$ a una humedad relativa promedio del $70 \%$ ). Este pretratamiento permite eliminar el exceso de mucílago que típicamente tiene el clon $\mathrm{CCN}-51$, el cual conduce a una fermentación que produce granos de cacao sin descriptores sensoriales especiales [12-14]. Seguidamente, las muestras se introducen en un cajón de masa total fermentable mayor de $200 \mathrm{~kg}$. La primera remoción del grano se hizo tras $48 \mathrm{~h}$ y siguieron remociones cada $24 \mathrm{~h}$ hasta finalizar el proceso (6 días en el cajón). El momento en que comenzó el escurrimiento se consideró el punto inicial de la fermentación (F0). Con el fin de inactivar la enzima polifenol oxidasa y evitar el avance de la fermentación y la oxidación, las muestras del nivel F0 fueron sumergidas en agua a $10-15^{\circ} \mathrm{C}$ durante $15 \mathrm{~min}$. Posteriormente, se separó el mucílago del grano de cacao y este último fue introducido en agua a $95^{\circ} \mathrm{C}$ durante $5 \mathrm{~min}$ [15]. Se realizó un análisis cualitativo frente a una muestra sin tratar (blanco) empleando una solución de $1,1 \mathrm{~g} / \mathrm{L}$ de catecol en ausencia de luz. Después de $5 \mathrm{~min}$ se verificó el grado de pardeamiento alcanzado por cada uno de los ensayos.

El secado se hizo en una casa elba con techo móvil, siguiendo el protocolo del Departamento de Investigación de FEDECACAO [11]; la humedad alcanzada después en cada uno de los niveles de secado (Tabla 1), se determinó empleando el método AOAC $931.0418^{\text {th }}$. Los resultados del porcentaje de humedad fueron usados con el fin de expresar el contenido de polifenoles totales en base seca. Cada muestra recolectada fue etiquetada de acuerdo al diseño experimental (Tabla 1) y se mantuvo en condiciones de refrigeración $\left(4^{\circ} \mathrm{C}\right)$, ausencia de luz y oxígeno reducido (empaque al vacío) hasta la realización de los análisis que se describen a continuación.

\section{Análisis del contenido de Polifenoles Totales $(P T)$ y Capacidad Antioxidante $(C A)$.}

Preparación de las muestras. Comprendió las etapas de desengrasado y extracción de polifenoles, según lo propuesto por Cadena y Herrera [16]. Un gramo (1g) de muestra (descascarillada y molida) fue desengrasado con $10 \mathrm{~mL}$ de $\mathrm{n}$-hexano en un baño ultrasónico 
(LC $30 \mathrm{H}$, Elma Ultrasonics, Singen, Alemania) por $15 \mathrm{~min}$ a $30^{\circ} \mathrm{C}$. Seguidamente, se centrifugó (equipo Heraeus Megafuge 16R, Thermo Fisher Scientific, Waltham, Massachusetts, USA) a 3500 rpm durante $15 \mathrm{~min}$ a $25^{\circ} \mathrm{C}$.

Este proceso se realizó tres veces. Para la extracción de polifenoles, la muestra desengrasada fue puesta en contacto con $20 \mathrm{~mL}$ de una solución etanol:agua $(80: 20 \mathrm{v})$ a $60^{\circ} \mathrm{C}$ y agitación constante durante $15 \mathrm{~min}$. Luego, fue centrifugada (utilizando los mismos parámetros empleados en el desengrasado) y el sobrenadante se pasó a través de papel filtro (papel cualitativo,

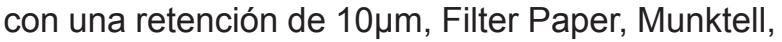
Bärenstein, Germany).

Laextracciónfuerealizadatresvecesyfinalmentelas fracciones filtradas fueron concentradas mediante evaporación a $50^{\circ} \mathrm{C}$ y $100 \mathrm{mbar}$ (rotoevaporador R-210, Büchi, Valinhos, Brasil). Cada extracto fue aforado a un volumen de $25 \mathrm{~mL}$ con una solución etanol:agua (50:50v) y almacenado a $4^{\circ} \mathrm{C}$ hasta su uso. Fueron preparados dos extractos por muestra recolectada. Algunos de ellos (uno por nivel de fermentación) fueron seleccionados y liofilizados para su posterior análisis por cromatografía líquida. El proceso de liofilización se realizó empleando un liofilizador SP Scientific, AdVantage Plus Freeze Dryers, modelo Wizard 2,0. Inicialmente, se desarrolló un secado primario a $233,15 \mathrm{~K}$ y $0,013 \mathrm{MPa}$ durante $36 \mathrm{~h}$, seguido de un y secado secundario en gradiente desde 263,15 hasta $318,15 \mathrm{~K}$ durante $24 \mathrm{~h}$.

Ensayo de Folin-Ciocalteu (FC). El contenido de Polifenoles Totales $(P T)$ de las muestras se determinó siguiendo el procedimiento descrito por Wollgast [17]. El ensayo fue hecho por duplicado a cada extracto. Brevemente, $50 \mu \mathrm{L}$ del extracto a valorar, $1,5 \mathrm{~mL}$ de reactivo de Folin-Ciocalteu (diluido 10 veces en agua destilada) y $1,5 \mathrm{~mL}$ de solución de $\mathrm{Na}_{2} \mathrm{CO}_{3}(7,5 \% \mathrm{p} / \mathrm{v})$ fueron mezclados vigorosamente. La mezcla fue dejada en reposo durante $60 \mathrm{~min}$ protegida de la luz. Luego, la absorbancia de las muestras fue leída a $765 \mathrm{~nm}$ en un espectrofotómetro (GENESYS 20, Thermo Spectronic, Waltham, Massachusetts, USA). EI contenido de $P T$ se expresó en mg equivalentes de ácido gálico (mgEAG) por gramo de muestra seca (gMS). Para la cuantificación del contenido de $P T$ se construyó una curva patrón (relación lineal entre la absorbancia y la concentración de la sustancia de referencia) en la que se empleó como sustancia de referencia el ácido gálico (AG). El procedimiento experimental empleado es idéntico al descrito anteriormente. La única diferencia es que en lugar de extracto a valorar se emplea la sustancia de referencia. Finalmente, el contenido de $P T$ fue calculado empleando la Ecuación 1 y expresado en $\mathrm{mg}$ equivalentes de ácido gálico (mgEAG) por gramo de muestra seca (gMS).

$$
P T=\frac{\left(A b s_{\text {muestra }}-b\right) / m}{g M s} \times 25 m l \times F
$$

En donde: $A b s_{\text {muestra }}$ representa la absorbancia de la muestra, $g M s$ representa el peso de la muestra (gramos), $m(1,748)$ representa la pendiente y $b(0,018)$ el intercepto de la curva patrón, $V$ es el volumen de aforo y $F$ factor de dilución de la muestra analizada.

\section{Ensayo ORAC (Oxygen Radical Absorbance} Capacity). La medición de la capacidad antioxidante $(C A)$ se desarrolló de acuerdo al método planteado por Huang et al. [18]. En los pozos de una microplaca oscura de 96 lugares se adicionaron los reactivos, en el orden que se especifica a continuación: $150 \mu \mathrm{L}$ de solución de fluoresceína $\left[8,16 \times 10^{-5} \mathrm{mM}\right], 25 \mu \mathrm{L}$ de Trolox® $[6,25-50 \mu \mathrm{M}]$, extracto $[0,0468-0,1238 \mathrm{mgEAG} / \mathrm{gMS}]$ o buffer de fosfato (según fuera a ubicarse en el pozo el estándar, la muestra o el blanco del ensayo, respectivamente) y $25 \mu \mathrm{L}$ de solución de AAPH [153mM]. Antes de añadir el AAPH, la microplaca con la fluoresceína y el antioxidante fue precalentada a $37^{\circ} \mathrm{C}$ durante $10 \mathrm{~min}$. Inmediatamente después de la adición del iniciador de radicales, se midió en un espectrofluorímetro (Fluoroskan Ascent, Thermo Scientific, Waltham, Massachusetts, USA) la señal de fluorescencia cada minuto durante $1 \mathrm{~h}$. Las longitudes de onda de excitación y emisión fueron 485 y 530nm, respectivamente. Cada concentración evaluada se analizó por triplicado (4 concentraciones por extracto). Los resultados se expresaron en $\mu \mathrm{mol}$ trolox-equivalente por gramo muestra seca ( $\mu \mathrm{molTE} / \mathrm{gMS})$.

Análisis de cromatografía líquida acoplada a masas (LC-MS-ESI-IT). La separación e identificación de los polifenoles presentes se hizo acorde al procedimiento desarrollado por Cienfuegos-Jovellanos et al. [19]. A partir de las muestras liofilizadas se prepararon soluciones de concentración $1 \mathrm{mg} / \mathrm{mL}$, que fueron pasadas 
a través de un filtro PTFE de $0,45 \mu \mathrm{m}$. Se realizó inyección directa (volumen de inyección: $20 \mu \mathrm{L}$ ) a un equipo de cromatografía líquida con detector UV-Vis (Elite LaChrom, VWR-HITACHI), adaptado a un espectrómetro de masas (Amazon $\mathrm{X}$, Bruker Daltonis, Ponte Alta - Atibaia, Brasil) con nebulización por electrospray (ESI) y analizador de trampa iónica (IT). Para la separación se utilizó una columna de fase reversa Zorbax Eclipse XDB-C18 $(150 \times 4,6 \mathrm{~mm}, 5 \mu \mathrm{m})$, a $25^{\circ} \mathrm{C}$. La fase móvil consistió en agua:ácido fórmico $(99,9: 0,1 \mathrm{v})$ (solvente A) y acetonitrilo (solvente B), a una velocidad de flujo de $0,8 \mathrm{~mL} / \mathrm{min}$. El gradiente fue el siguiente: 0-20min, 6-10\% B lineal; 20-25min, $10-13 \%$ B lineal ; 25-30min, 13-15\% B lineal; 3040min, 15-10\% B lineal; 40-45min, 10-6\% B lineal, seguido de $10 \mathrm{~min}$ de re-equilibrio de la columna antes de una nueva inyección. El detector del espectrómetro de masas se operó en modo full scan negativo; la inyección a la fuente de iones del electrospray se efectuó con un splitting del $40 \%$.

En la trampa de iones, la temperatura y el voltaje del capilar fueron $355^{\circ} \mathrm{C}$ y $-6 \mathrm{~V}$. El voltaje del spray fue de $4,7 \mathrm{kV}$. Los cromatogramas se registraron a $280 \mathrm{~nm}$. La identificación de las sustancias presentes se hizo tomando como base los espectros de masas (iones pseudomoleculares de los compuestos de interés) y el orden de elución en el perfil cromatográfico y comparación con estándares.

\section{Análisis estadístico}

La influencia de los tiempos de fermentación y secado sobre el contenido de $P T$ y la $C A$ fue estudiada empleando el diseño experimental factorial multinivel que se muestra en la Tabla 1. El análisis estadístico se desarrolló empleando el software STATGRAPHICS Centurion XVI (Trial version). Las diferencias significativas entre los valores promedio obtenidos fueron determinadas mediante la prueba Tukey HSD. A través de un análisis de varianza se establecieron los factores con efecto significativo sobre las dos variables de respuesta.

Tabla 1. Factores y niveles del diseño experimental.

\begin{tabular}{|c|c|c|c|c|c|c|c|}
\hline \multirow{2}{*}{ Factores } & \multirow{2}{*}{ Símbolo } & \multirow{2}{*}{ Unidades } & \multicolumn{5}{|c|}{ Nivel } \\
\hline & & & 1 & 2 & 3 & 4 & 5 \\
\hline $\begin{array}{l}\text { Tiempo de } \\
\text { fermentación }\end{array}$ & $\mathrm{F}$ & Días & 0 & 2 & 4 & 6 & 8 \\
\hline $\begin{array}{l}\text { Tiempo de } \\
\text { secado }\end{array}$ & $S$ & Días & 1 & 3 & 5 & -- & -- \\
\hline
\end{tabular}

\section{Resultados}

\section{Contenido de Polifenoles Totales (PT)}

En la Figura 1 se muestra el efecto de los días de fermentación y secado sobre el contenido de $P T$ del clon de cacao CCN-51. La gráfica obtenida puede ser dividida en tres regiones con tendencias bien definidas en lo que se refiere a la evolución de los $P T$ durante el proceso de beneficio del clon analizado: inicial (F0 y F2), intermedia $(\mathrm{F} 4)$ y final ( $F 6$ y $F 8)$, con valores promedio de $73,48 \pm 4,93,47,85 \pm 6,46$ y $36,68 \pm 2,08 \mathrm{mgEAG} / \mathrm{gMS}$, respectivamente.

De acuerdo con el análisis de varianza, los días de fermentación tienen un efecto significativo sobre el contenido de $P T$ del clon $\mathrm{CCN}-51$ con un nivel de confianza del $95 \%$.

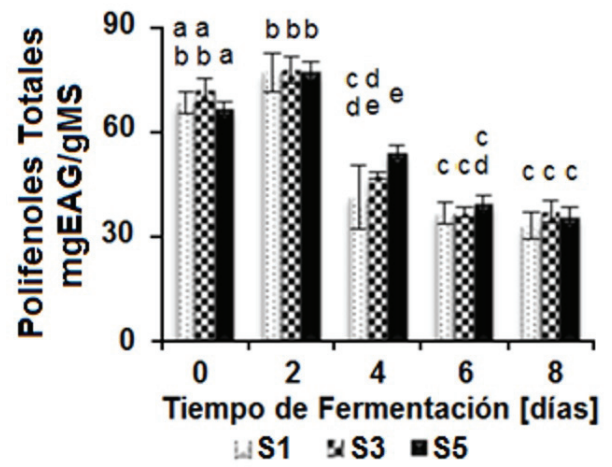

Figura 1. Variación del contenido de polifenoles totales durante la fermentación (F) y el secado $(S)$ del clon de cacao CCN-51. Los resultados se expresan como la media \pm desviación estándar para $n=8$. S1, S3 y S5 representan 1, 3 y 5 días de secado, respectivamente. Las letras (a,b,c,d,e) expresan diferencias significativas entre medias con un nivel de confianza $p<0,05$.

El diagrama de Pareto de la Figura 2 esquematiza los resultados del ANOVA, evidenciando la incidencia negativa de esta etapa del beneficio en la variable de respuesta analizada. El secado, por su parte, no posee efecto significativo en el contenido de $P T$.

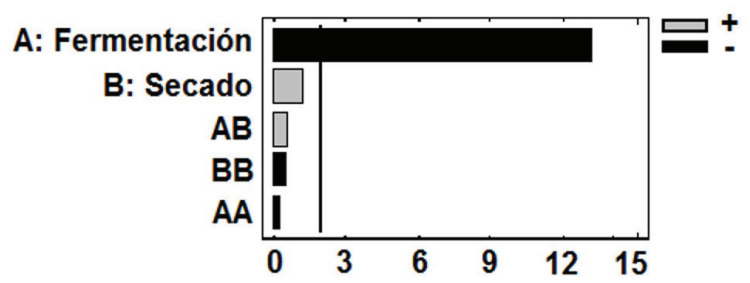

Figura 2. Diagrama de Pareto estandarizado de la influencia de la fermentación y del secado sobre el contenido de polifenoles totales del clon de cacao CCN-51. 
Una vez establecido que los días de fermentación son la variable relevante durante el proceso de beneficio del clon CCN-51, la evolución del contenido de $P T$ pudo ajustarse a un modelo matemático para predecir el comportamiento observado.

El ajuste tomó en cuenta los datos experimentales a partir del segundo día de fermentación, dado que aquellos obtenidos para las muestras sin fermentar estuvieron muy por debajo de lo esperado y/o reportado en la bibliografía relacionada. Se propuso un modelo unifactor del tipo recíproco (Ecuación 2) para explicar la variación del contenido de $P T$ en función del tiempo de fermentación $(T F)$.

$$
P T=\frac{1}{0,007142+\left(0,003025 \times T F^{1,0055}\right)}
$$

El coeficiente de correlación ajustado $\left(R^{2}\right)$, considerando el conjunto de datos experimentales sobre los cuales se hizo el ajuste $(2 \leq T F \leq 8)$, fue de 0,963 . En la Figura 3 puede apreciarse el grado de coincidencia presentado entre el modelo propuesto y los datos experimentales.

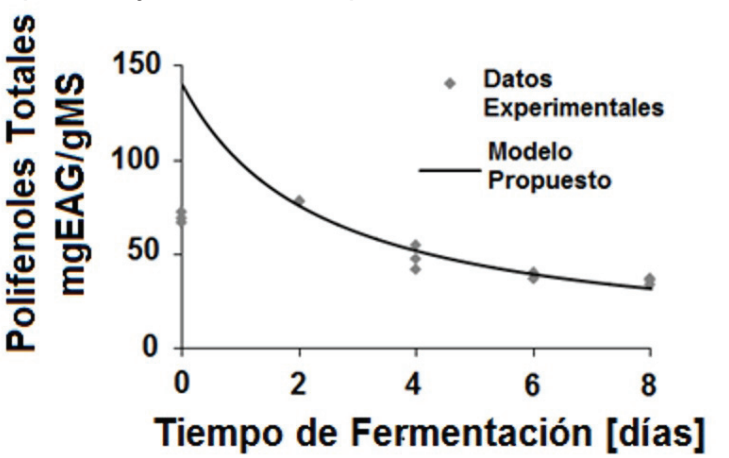

Figura 3. Grado de ajuste a los datos experimentales del modelo propuesto para predecir la evolución del contenido de polifenoles totales en función del tiempo de fermentación.

\section{Capacidad antioxidante $(C A)$}

En el caso de la $C A$ se determinó que, de forma similar a como ocurre con el contenido de $P T$, la fermentación es el factor que genera mayor incidencia. En general, a medida que avanza la fermentación del clon $\mathrm{CCN}-51$, la $C A$ disminuye. Los valores obtenidos se presentan en la Figura 4, expresados en $\mu \mathrm{molTE} / \mathrm{gMS}$.

$\mathrm{Al}$ igual que como en el caso de los polifenoles totales, la variación de $C A$ puede dividirse en tres etapas en función del proceso de beneficio: inicial (F0y F2), intermedia(F4)y final (F6y F8), convalores ORAC promedio de $969,42 \pm 68,44,593,24 \pm 110,06$ y $404,20 \pm 53,00 \mu \mathrm{molTE} / \mathrm{gMS}$, respectivamente. En el diagrama de Pareto presentado en la Figura 5 se observa que la fermentación y el secado tienen incidencia significativa sobre la variable de respuesta con un nivel de confianza de $95 \%$. El proceso fermentativo posee un efecto negativo sobre el valor de $C A$, mientras que el efecto del secado no es significativo a excepción de F4 (entre S1 y S3-S5).

Aunque según el ANOVA ambas etapas del beneficio del clon $\mathrm{CCN}-51$ afectan de forma significativa el valor de la $C A$, pudo inferirse que el secado tiene un impacto mínimo sobre la variable de respuesta. Esto, luego de comparar la relación $F \quad\left(\sigma_{\text {intergrupos }} / \sigma_{\text {intragrupos }}\right)$ de este factor $(F=8,12)$ respecto a la de la fermentación $(F=257,79)$.

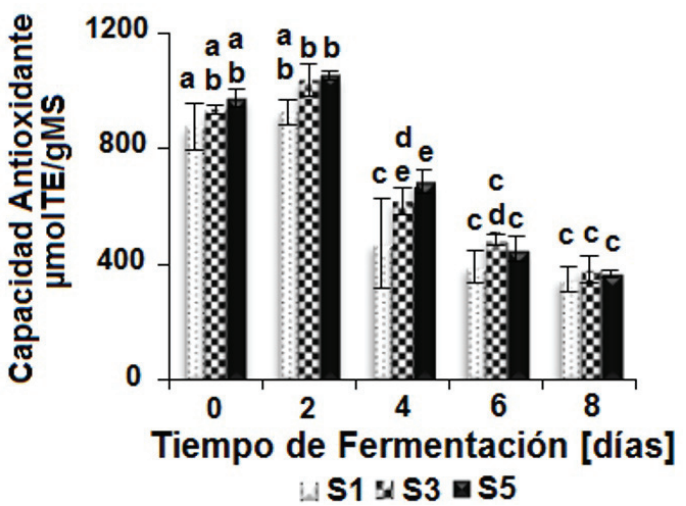

Figura 4. Variación de la capacidad antioxidante durante la fermentación (F) y el secado (S) del clon de cacao CCN-51. Los resultados se expresan como la media \pm desviación estándar para $n=8$. Las letras

(a,b,c,d,e) expresan diferencias significativas entre medias con un nivel de confianza $p<0,05$.

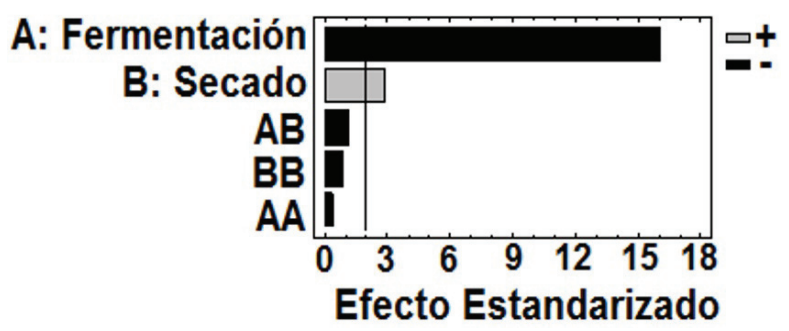

Figura 5. Diagrama de Pareto estandarizado de la influencia de la fermentación y del secado sobre la capacidad antioxidante del clon de cacao CCN-51. 
El resultado obtenido permitió verificar que existe una correlación inversa entre la $C A$ y el grado de fermentación del clon CCN-51. Por el contrario, la variación en los días de secado dio lugar a valores de $C A$ que oscilaron en un intervalo de valores prácticamente constante, es decir, se confirmó la existencia de una correlación nula entre la $C A$ del clon CCN-51 y este factor. Nuevamente se ajustó un modelo matemático unifactor recíproco para relacionar la $C A$ del clon $\mathrm{CCN}-51$ con la variable del beneficio que tiene la mayor incidencia sobre su variación: la fermentación (Ecuación 3). Los datos experimentales considerados para tal ajuste, en congruencia con lo expresado en el ítem de polifenoles totales, fueron aquellos recolectados del día 2 al 8 de fermentación.

$$
C A=\frac{1}{0,000569+0,000179 \times T F^{1,2502}}
$$

El coeficiente de correlación ajustado $\left(R^{2}\right)$, calculado en el intervalo $2 \leq T F \leq 8$, fue de 0,959.

En la Figura 6 puede apreciarse el grado de ajuste del modelo propuesto, al igual que los datos experimentales.

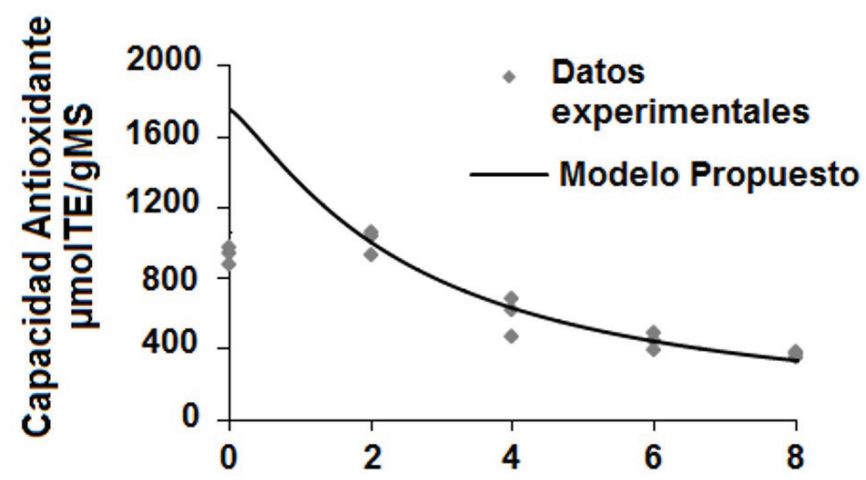

Figura 6. Grado de ajuste a los datos experimentales del modelo propuesto para predecir el comportamiento de la capacidad antioxidante en función del tiempo de fermentación del clon de cacao CCN-51.

Cuando se evalúa el tipo de relación existente entre el contenido de $P T$ y de la $C A$, el resultado es una correlación lineal directa, con un grado de ajuste $\left(R^{2}\right)$ de 0,95 (Figura 7 ). Esta tendencia no sólo es propia del cacao, ella ha sido observada en otro tipo de matrices [1, 20-22].

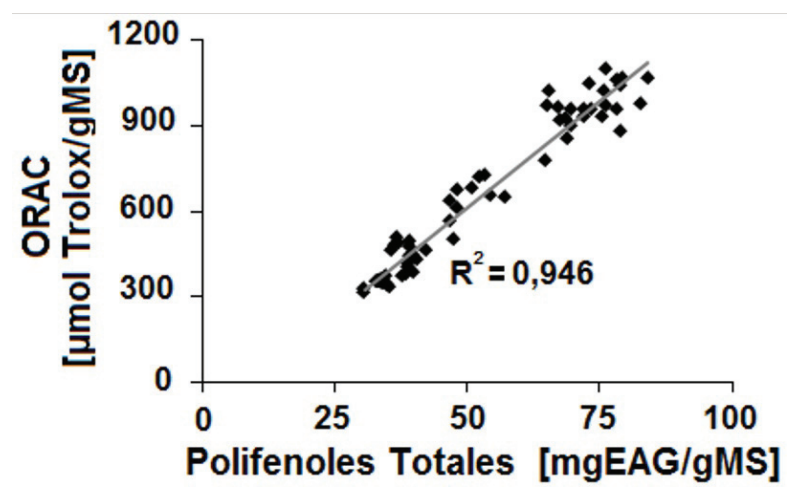

Figura 7. Relación entre la cantidad de polifenoles totales y la capacidad antioxidante en el clon de cacao CCN-51.

\section{Separación e identificación de sustancias polifenólicas}

Para la realización de esta prueba fueron seleccionadas cuatro muestras del diseño experimental: todas al mismo nivel de secado ( 5 días), del día 2 a 8 de fermentación. En la Figura 8 se muestra el perfil cromatográfico obtenido para una muestra con 2 días de fermentación y 5 días de secado. En cada uno de los extractos analizados fueron obtenidas 11 señales bien definidas análogas a los mostrados en la Figura 8. La identificación tentativa de las sustancias se hizo comparando el espectro de masas $(\mathrm{m} / \mathrm{z}$ del ion pseudomolecular de carga simple o múltiple) con lo reportado en la literatura en estudios similares [22-25]. Además, se tuvo en cuenta el orden de elución de cada compuesto en el perfil cromatográfico. Los resultados del análisis por espectroscopía de masas se muestran en la Tabla 2. 
Tabla 2. Compuestos fenólicos tentativamente identificados por LC-MS en el clon de cacao CCN-51.

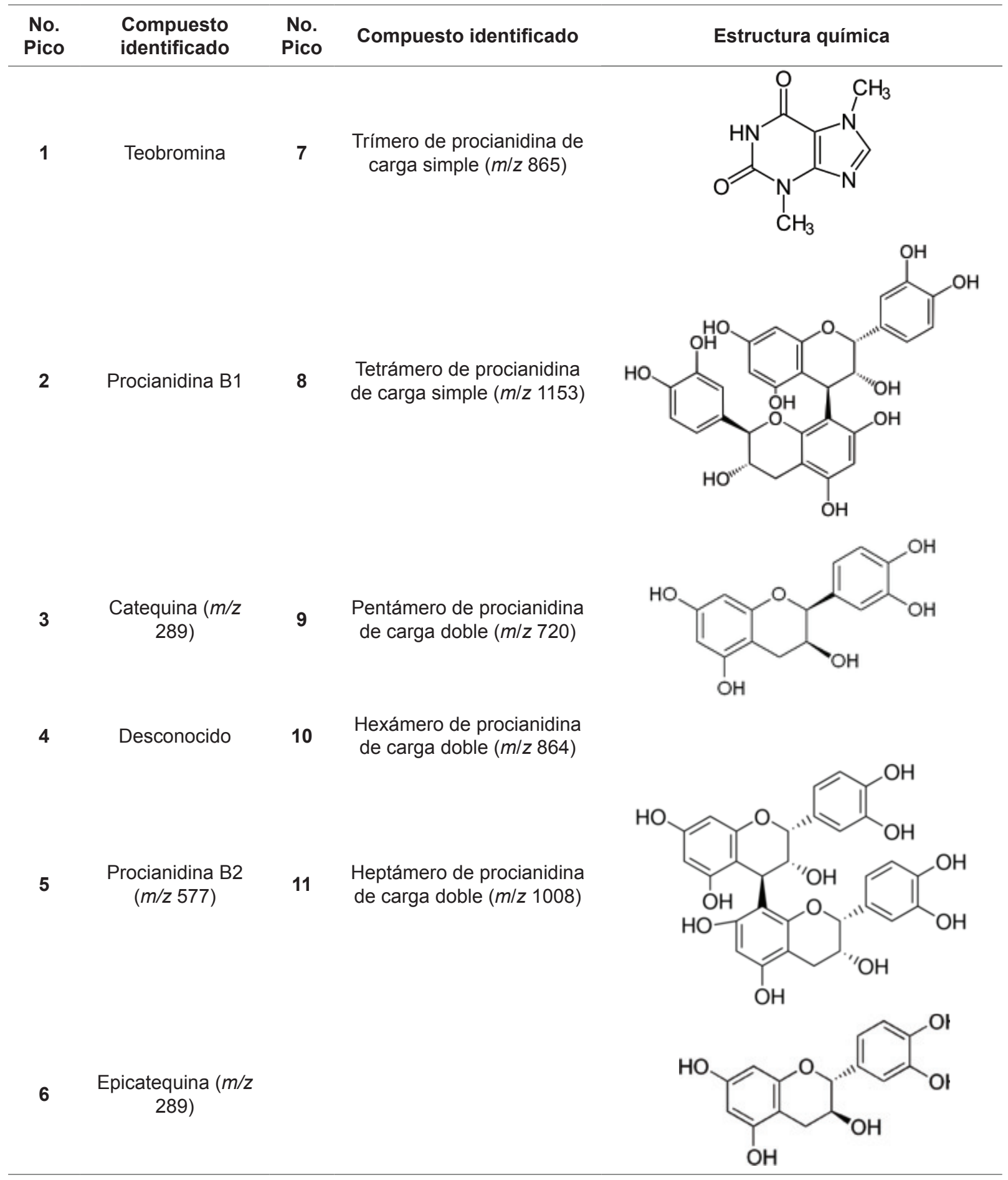




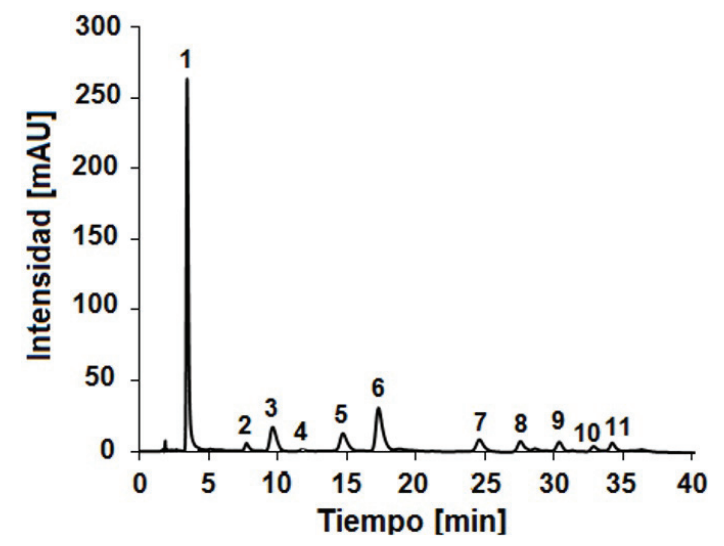

Figura 8. Perfil cromatográfico (HPLC) de la muestra F2S5. Ver Tabla 2 para la identificación de las señales.

Luego de identificar las sustancias antioxidantes presentes en los extractos, se evaluó cualitativamente su variación durante el proceso fermentativo. Se tomó como referencia el nivel 2 y a partir de éste se calcularon valores relativos en los demás niveles. La Figura 9 muestra el comportamiento seguido por los monómeros (catequina y epicatequina), dímeros y oligómeros (trímeros a heptámeros) hallados en las muestras. Todos los flavonoides identificados, a excepción de la procianidina B1 y la catequina, siguieron una tendencia de reducción en los niveles evaluados; dentro de cada nivel de fermentación la cantidad relativa de procianidina $\mathrm{B} 2$, epicatequina $\mathrm{y}$ sus oligómeros permaneció aproximadamente constante (Figura 9). Los mayores valores relativos se presentaron en el segundo día de fermentación y fueron disminuyendo progresivamente con el avance del proceso hasta el día seis. La reducción del día 2 al 4 fue del $35 \%$, con un valor relativo de $0,65 \pm 0,03$. Por su parte, la relación F6/F2 se ubicó alrededor de $0,40 \pm 0,03$; la disminución fue del $60 \%$. La muestra correspondiente al nivel F8 mostró valores de área inferiores a la muestra F2 en un 65\%; en este caso el valor relativo se fijó en $0,34 \pm 0,05$. Entre el sexto y el octavo día de fermentación el porcentaje de desviación relativa fue inferior a $15 \%$. Salvo por la muestra correspondiente al cuarto día de fermentación, la catequina y la procianidina B1 presentaron valores relativos constante y superior, respectivamente, en las muestras evaluadas. El aumento relativo del contenido de procianidina B1 para el cuarto día de fermentación no es consistente con los resultados discutidos en el trabajo de Wollgast y Anklam [6] en donde se evidencia una disminución del $42,62 \%$ en el contenido de procianidinas totales en 4 días de fermentación; sin embargo, los resultados de reducción relativa de procianidinas al final de la fermentación obtenidos en este trabajo (alrededor del $65 \%$ ), son cercanos a los discutidos por Wollgast y Anklam [6] (alrededor del 54\%).

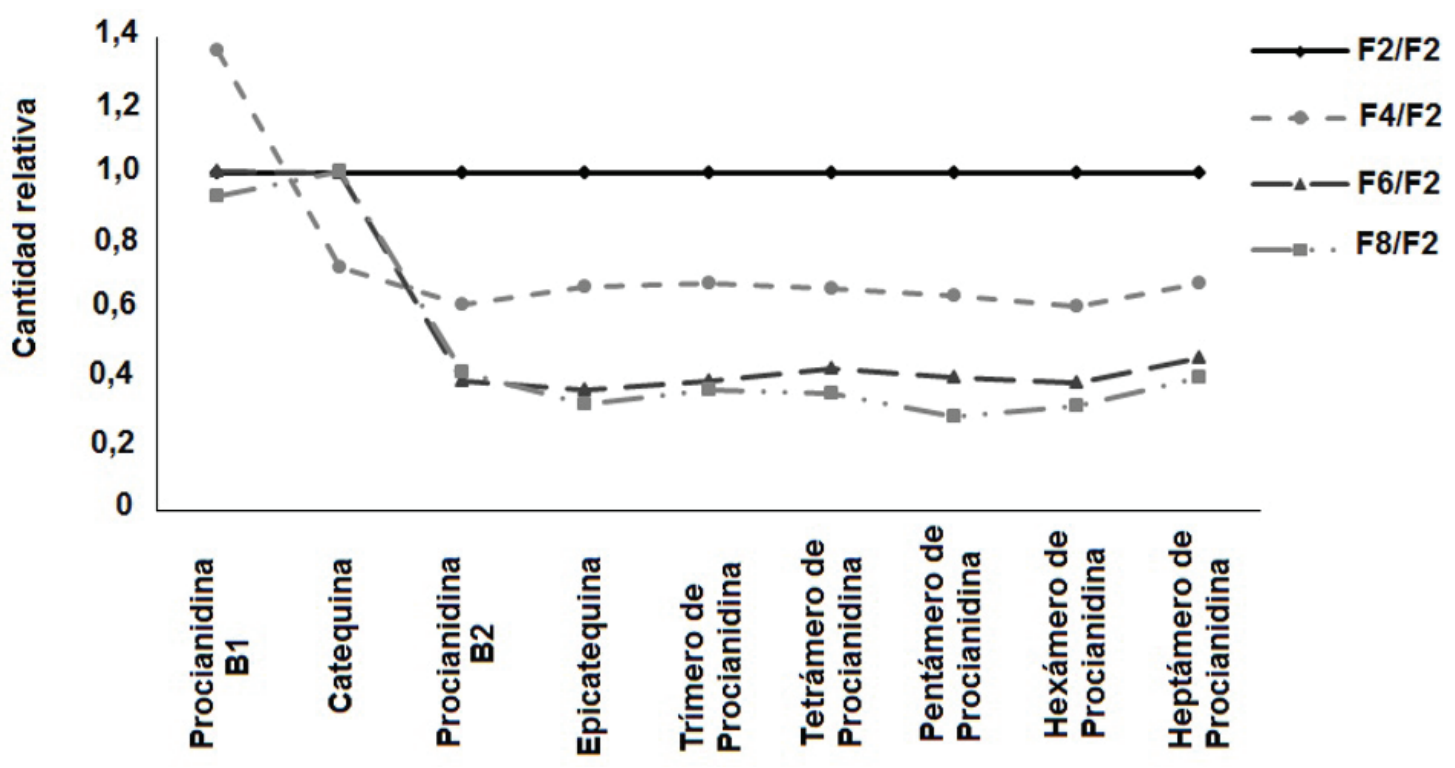

Figura 9. Valores relativos de las sustancias antioxidantes presentes en el clon de cacao CCN-51, respecto al nivel F2. 


\section{Discusión}

\section{Contenido de polifenoles totales (PT)}

Evolución durante el beneficio. Un análisis general de la Figura 1 permite establecer que la fermentación genera una disminución pronunciada del contenido de $P T$, mientras que el secado no posee efecto definido.

Durante la fermentación del grano de cacao, los polifenoles que están almacenados en las células pigmentadas de los cotiledones, también llamadas células de almacenamiento de polifenoles, son liberados junto con otros líquidos celulares. Según Wollgst y Anklam [6], durante los primeros dos días de la fermentación, el mucílago adherido a los granos se desprende y existe un aumento de temperatura. Bajo condiciones anaeróbicas los microorganismos producen ácido acético y etanol. Estos procesos inhiben la germinación del grano y contribuye a los cambios estructurales en los granos fermentados, entre los que se destacan la remoción de enzimas y sustratos. Los líquidos celulares se mueven y atraviesan las paredes celulares y se distribuyen sobre todo el grano de cacao. Para el tercer día, la masa de granos se habrá calentado uniformemente a $45^{\circ} \mathrm{C}$ y se mantendrá entre esta temperatura y $50^{\circ} \mathrm{C}$ hasta que la fermentación se haya completado.

Numerosos estudios han reportado la proporcionalidad inversa del contenido de $P T$ y el tiempo de fermentación del cacao, que se reconoce (junto al tostado) como una de las etapas del procesamiento en que ocurre la mayor pérdida de sustancias antioxidantes $[1,3,8,26]$. Así mismo, estudios bibliográficos han concluido que la menor disminución de $P T$ ocurre durante el secado natural $[27,28]$.

Etapa inicial (FO y F2): En esta región se ubicaron las muestras con los contenidos más altos de $P T$. No obstante, los valores obtenidos en los granos sin fermentar (F0) se encuentran por debajo de aquellos reportados en estudios similares. Así por ejemplo, Tomas-Barberán et al. [29] hallaron un contenido polifenólico total de 81,4mgEAG/gMS en un clon amazónico colombiano seco y sin fermentar. Kim y Keeney [30] encontraron contenidos de $P T$ en granos de cacao forastero no fermentado entre 120 y $180 \mathrm{mgEAG/gMS}$. Afoakwa et al. [31] midieron el contenido de $P T$ en híbridos de la variedad forastero y obtuvieron un valor de 180,87mgEAG/gMS. Por su parte, Serra Bonheví y Ventura [32] y Misnawi et al. [33] reportaron valores de $P T$ de 135mgEAG/ gMS y $140 \mathrm{mgEAG} / \mathrm{gMS}$ respectivamente, en granos de cacao fresco.

Adicionalmente, los polifenoles presentan reacciones de oxidación que producen taninos condensados insolubles de alto peso molecular [3]. Dichas reacciones son catalizadas por la enzima polifenol oxidasa (PPO), con gran actividad en los granos en estado fresco. El hecho que el contenido de $P T$ de los granos de CCN-51 no fermentados haya resultado inferior al reportado en varias fuentes bibliográficas, puede atribuirse a que el procedimiento realizado para inactivar esta enzima no haya sido suficiente para inhibirla al $100 \%$. Esta hipótesis de trabajo se sustenta en el trabajo realizado por Godoy [34], quien propone un método en el cual se optimizan las condiciones que influyen en el proceso de inhibición (temperatura del tratamiento térmico y concentración de inhibidor). Entre los resultados más sobresalientes de Godoy [31], se destacan el ajuste de un modelo que correlaciona el porcentaje de inhibición de la PPO y la recuperación de PT. Así mismo con base en las condiciones optimizadas, se alcanza una inhibición superior al $90 \%$ para un clon de cacao ICS91. Sin embargo, debe mencionarse que la tendencia seguida por el contenido de $P T$ en el presente estudio durante los dos primeros días de fermentación, es concordante con lo reportado por Gil [35].

En el trabajo de dicho autor se evidencia un ligero aumento de la variable analizada entre el primer y el segundo día de fermentación. Este aumento sugiere que las primeras reacciones tanto químicas como enzimáticas del proceso fermentativo convierten algunos de los polifenoles a formas más reductoras y activas al ensayo de Folin-Ciocalteu.

$\mathrm{Se}$ sugiere entonces para investigaciones posteriores, la realización de análisis más específicos a las muestras obtenidas en los primeros días de fermentación (p. ej. HPLC), con el fin de establecer con total certeza el contenido real de $P T$.

Etapa intermedia (F4): Fue la única que presentó variación con el factor secado, exhibiendo cambios significativos dentro del grupo. Específicamente, para la muestra correspondiente a cuatro días de fermentación y un día de secado (F4S1) se obtuvieron valores de $P T$ por debajo del promedio del grupo, comparables con aquellos de la etapa final de fermentación. 
Etapa final (F6 y F8): En esta parte del proceso el contenido de $P T$ dejó de ser dependiente de los días de fermentación. Este hecho coincide con lo reportado por Serra Bonheví et al. [32], los cuales encontraron que en el intervalo de 5-6 días del proceso de fermentación los $P T$ llegaban a un valor estable de $43,1 \pm 1,87 \mathrm{mgEAG} / \mathrm{gMS}$. Durante su estudio con frutos de cacao tipo Forastero, Efraim et al. [27] encontraron una disminución de polifenoles del $59 \%$ al finalizar el séptimo día de fermentación.

Así mismo, estos autores concluyeron que la etapa de secado al sol sólo disminuye en un $2,8 \%$ el contenido de $P T$ de semillas totalmente fermentadas. En el presente estudio los $P T$ se redujeron en un $50,1 \%$ al pasar del 1 er al 8 vo día de fermentación, concordando con los resultados de estudios previos $[1,3,8,26]$.
Modelo matemático. Se observa que el valor predicho para el nivel 0 de fermentación se aleja considerablemente del contenido de $P T$ establecido en el presente estudio, acercándose a su vez a lo reportado por otros autores [29-32].

Aunque la expresión propuesta en este trabajo fue ajustada con valores propios del clon de cacao CCN-51, ella también permite predecir la evolución del contenido de $P T$ de variedades Forastero y Amazónico con un error relativo promedio del $20 \%$, aproximadamente (Tabla 3 ). Incrementar el poder predictivo de la ecuación planteada, así como extender su utilización para el cálculo de $P T$ en otras variedades de cacao puede lograrse mediante la obtención de datos experimentales en clones diferentes al CCN-51 que posteriormente serán utilizados en el robustecimiento de los valores de las constantes aquí calculadas.

Tabla 3. Error relativo (ER) porcentual de $P T$ reportados por otros autores y $P T$ calculados con la ecuación planteada, para diferentes muestras de cacao.

\begin{tabular}{cccccc}
\hline $\begin{array}{c}F \\
\text { [días] }\end{array}$ & $\begin{array}{c}\text { Tipo } \\
\text { Cacao }\end{array}$ & $\begin{array}{c}\boldsymbol{P T} \text { [mgEAG/ } \\
\text { gMS] } \\
\text { reportado }\end{array}$ & $\begin{array}{c}\boldsymbol{P T} \text { [mgEAG/ } \\
\text { gMS] } \\
\text { calculado }\end{array}$ & ER [\%] & Fuente \\
\hline 0 & Forastero & 120,00 & 140,02 & 14 & Kim y Keneey 1984 [30] \\
0 & no reportado & 135,00 & 140,02 & 4 & Serra y Ventura 1997 [32] \\
5 & no reportado & 43,10 & 44,64 & 3 & Serra y Ventura 1997 [32] \\
0 & Amazónico & 81,40 & 140,02 & 42 & Tomás-Barberan et al., 2007 [29] \\
0 & Forastero & 108,76 & 140,02 & 22 & Efraim et al., 2010 [27] \\
3 & Forastero & 71,42 & 61,46 & 16 & Efraim et al., 2010 [27] \\
7 & Forastero & 44,55 & 35,03 & 27 & Efraim et al., 2010 [27] \\
0 & híbrido forastero & 180,87 & 140,02 & 29 & Afoakwa et al., 2012 [31] \\
\hline & & & ER Promedio & $\mathbf{2 0}$ &
\end{tabular}

\section{Capacidad Antioxidante ( $C A$ )}

Evolución durante el beneficio. Las etapas que describen la variación de la $C A$ durante el beneficio del clon CCN-51 pueden ser analizadas de forma similar al contenido de $P T$ : una etapa inicial integrada por los niveles 0 y 2 de fermentación, caracterizada por los valores más altos de $C A$ y sin efecto pronunciado de la variable secado.

Seguida de una fase intermedia, correspondiente al cuarto día de fermentación, en la que se observa incidencia significativa del secado en los valores de $C A$. Por último, una etapa final que agrupa los niveles 6 y 8 de fermentación, sin presencia de efecto del secado y en la que el valor de $C A$ se estabiliza.

El promedio obtenido para la $C A$ de todas las muestras evaluadas, determinada por el método ORAC, fue de $668,09 \pm 272,70 \mu \mathrm{molTE} / \mathrm{gMS}$. La desviación estándar de 272,70 se traduce en un coeficiente de variación de $40,82 \%$, el cual se explica si se considera que los valores de $C A$ variaron entre $969,42 \pm 68,44$ y $404,20 \pm 53,00 \mu \mathrm{molTE} / \mathrm{gMS}$ para el inicio y final de la fermentación.

En el caso de cacao sin procesamiento industrial, Gil [35] encontró valores de ORAC de aproximadamente $450 \mu \mathrm{molTE} / \mathrm{gMS}$ para muestras con 5 días de fermentación y 5 días de secado en cacao colombiano, lo cual es equivalente con los resultados de $C A$ obtenidos para la muestra F6S5 $(451,43 \pm 43,16 \mu \mathrm{molTE} / \mathrm{gMS})$.

Modelo matemático. En el ajuste de la expresión propuesta en este trabajo se utilizaron valores propios del clon de cacao CCN-51. Sin embargo, se verificó su capacidad de predicción de la $C A$ en otras variedades de cacao. Para tal fin, los valores 
de CA hallados por Gil [35] en su análisis de cacao colombiano con diferentes días de fermentación, se compararon con aquellos predichos por la ecuación planteada, obteniendo un error relativo porcentual del $20 \%$ (Tabla 4). Así las cosas, se concluye que es posible usar la ecuación ajustada para predecir la $C A$ de clones de cacao diferentes al CCN-51, así como mejorar su nivel predictivo e incrementar su alcance mediante experimentación con otras variedades de cacao.

Tabla 4. Error relativo (ER) porcentual de la capacidad antioxidante (CA) para cacao colombiano (fuente: Gil, 2012 [35]) y la CA calculada con la ecuación propuesta en el presente trabajo.

\begin{tabular}{|c|c|c|c|}
\hline Fermentación [días] & $\begin{array}{c}\text { CA [ } \mu \text { molTE/gMS] } \\
\text { Reportado }\end{array}$ & $\begin{array}{c}\text { CA [ } \mu \text { molTE/gMS }] \\
\text { Calculado }\end{array}$ & ER [\%] \\
\hline 2 & 920,00 & 1005,23 & 8,5 \\
\hline 3 & 750,00 & 783,77 & 4,3 \\
\hline 4 & 740,00 & 632,17 & 17,0 \\
\hline 5 & 800,00 & 524,17 & 52,6 \\
\hline \multicolumn{3}{|c|}{ Promedio ER } & 20,6 \\
\hline
\end{tabular}

Debe resaltarse que los resultados de $C A$ reportados por Gil [35] no son del todo concordantes con los reportados en el presente estudio.

Gil [35] reporta una disminución del valor de $C A$ hasta el cuarto día de fermentación, seguido de un incremento en el día quinto de dicho proceso. Ese incremento concuerda con los valores de $P T$ hallados por el autor mencionado, que también aumentan entre el cuarto y quinto día del proceso fermentativo.

Tal como se observa en la Tabla 4, este hecho hace que se generen los mayores porcentajes de error relativo entre el valor calculado y el reportado por el Gil [32]. Ante lo cual se propone que los experimentos posteriores (conducentes a incrementar el nivel predictivo del modelo planteado) hagan especial énfasis en la parte final del proceso de fermentación.

\section{Relación entre la capacidad antioxidante y el contenido de polifenoles totales}

El contenido de $P T$ y la $C A$ han sido relacionados previamente por diferentes autores. Adamson et al. [36] y Gu et al. [2] reportaron una relación lineal entre la $C A$ y el contenido de procianidinas, para diferentes productos de cacao, con $\mathrm{R}^{2}$ de 0,994 y 0,923, respectivamente. Miller et al. [37] correlacionaron la $C A$ determinada por el método ORAC con el contenido de sólidos de cacao libres de grasa (NFCS de sus siglas en inglés) para diferentes productos de chocolate y encontraron un $R^{2}$ de 0,985 . A su vez, el contenido de NFCS fue directamente proporcional al contenido de $P T\left(\mathrm{R}^{2}=0,979\right)$. En su trabajo con licor de cacao criollo, trinitario, forastero y nacional, Counet et al. [38] encontraron una correlación positiva entre el nivel de procianidinas y el tiempo de inhibición de la oxidación por el método ORAC $\left(R^{2}=0,936\right)$. Los resultados obtenidos en este estudio son comparables con los reportados por los autores mencionados, teniendo en cuenta que las procianidinas forman parte de los polifenoles típicos del cacao [38].

\section{Separación e identificación de sustancias polifenólicas}

La gran mayoría de sustancias presentes en los extractos analizados pertenecen al grupo de los flavonoides, metabolitos secundarios de las plantas y uno de los grupos más representativos de los polifenoles [3]. Los granos de cacao son una fuente concentrada de polifenoles, donde los flavan-3-oles y sus derivados presentan las mayores concentraciones [31].

En las muestras analizadas estuvieron presentes los flavan-3-oles (+)-catequina y (-)-epicatequina (monómeros) y las procianidinas B1 y B2 (dímeros). Así mismo, se identificaron procianidinas en formas oligoméricas. Estudios previos de varios autores han demostrado que los flavanoles y dímeros ya mencionados son los más abundantes en el cacao y sus derivados. Además, las procianidinas halladas son características del cacao y del chocolate [39]. Estas últimas están constituidas por unidades de flavanoles (epicatequina principalmente) con enlaces $4 \rightarrow 6$ o $4 \rightarrow 8$ formando una mezcla de trímeros, tetrámeros y polímeros de hasta 10 unidades [24,25,39-40]. Tanto los flavanoles como las procianidinas han sido relacionados con las propiedades antioxidantes atribuidas al cacao. 
La variación de sustancias individuales a lo largo de la fermentación permite asegurar que la $C A$ de las muestras evaluadas se encuentra fuertemente influenciada por su contenido de epicatequina. El otro monómero, catequina, no parece influenciar significativamente dicha variable. Al respecto, Schroeter et al. [41] han reportado que el contenido de epicatequina es la principal razón de los beneficios en la salud vascular asociados al cacao y al chocolate. Así mismo, aseguran que la elección de un grano fermentado con alto contenido en polifenoles (y por ende alta capacidad antioxidante, dada la relación lineal existente entre $P T$ y $C A$ ) depende en gran medida de su contenido de epicatequina.

\section{Conclusiones}

De las etapas de beneficio del cacao, la fermentación es la que mayor impacto genera en la variación del contenido de polifenoles totales y la capacidad antioxidante del clon de cacao CCN-51. En ambos casos se observó un comportamiento decreciente en relación con el proceso fermentativo (disminución de $50,1 \%$ en el contenido de $P T$ y de $58,3 \%$ en la $C A$ ). El análisis de resultados también permitió determinar, con un nivel de confianza de $95 \%$, que el secado natural tiene un efecto poco significativo en las variables de respuesta seleccionadas. Teniendo en cuenta este hecho, se establecieron dos modelos unifactor de tipo recíproco para predecir el comportamiento de los polifenoles totales y la capacidad antioxidante en función del tiempo de fermentación del clon $\mathrm{CCN}-51$. Las ecuaciones planteadas presentaron un buen grado de correlación $\left(R^{2}=0,963\right.$ para $P T$ y $\mathrm{R}^{2}=0,959$ para $C A$ ) y fueron además satisfactoriamente aplicadas a la evaluación del proceso fermentativo en clones de cacao de tipo Forastero y Amazónico, con un porcentaje de error relativo promedio de aproximadamente $20 \%$. En el análisis por HPLC se identificaron los polifenoles presentes en las muestras evaluadas. La evaluación de su variación a través del proceso fermentativo permitió establecer que el avance de esta etapa del beneficio ocasiona una disminución en la mayoría de monómeros y oligómeros presentes (excepto catequina y procianidina B1), que se traduce a su vez en una disminución de la capacidad antioxidante de las muestras evaluadas. Dicha disminución está relacionada principalmente con el contenido del monómero epicatequina. El contenido de polifenoles totales y la capacidad antioxidante del clon evaluado presentaron un tipo de correlación directamente proporcional $\left(\mathrm{R}^{2}=0,95\right)$.

La utilización comercial de los extractos polifenólicos obtenidos a partir del clon no fermentado no queda reducida a la industria de alimentos, sino que incluye también las industrias cosmética y farmacéutica.

\section{Agradecimientos}

Los autores de este trabajo agradecen a la Vicerrectoría de Investigación y Extensión por el apoyo económico ofrecido a través del proyecto 5466 y 1318. Así mismo, los autores agradecen a Colciencias por el apoyo recibido a través del programa de jóvenes investigadores e innovadores.

\section{Referencias bibliográficas}

[1] Payne M, Hurst J, Miller K, Rank C, Stuart D. Impact of fermentation, drying, roasting and dutch processing on epicatechin and catechin content of cacao beans and cocoa ingredients. J Agric Food Chem. 2010;58(19):10518-27.

[2] Gu L, House S, Wu X, Ou B, Prior R. Procyanidin and catechin contents and antioxidant capacity of cocoa and chocolate products. J Agric Food Chem. 2006;54(11):4057-61.

[3] Wollgast J, Anklam E. Review on polyphenols in Theobroma cacao: changes in composition during the manufacture of chocolate and methodology for indentification and quantification. Food Res Int. 2000;33(6):423-47.

[4] Othman A, Ismail A, Ghani N, Adenan I. Antioxidant capacity and phenolic content of cocoa beans. Food Chem. 2007;100(4):1523-30.

[5] Afoakwa E. Chocolate science and technology. 1st ed. New Delhi: John Wiley \& Sons Ltd; 2010.

[6] Wollgast J, Anklam E. Polyphenols in chocolate: is there a contribution to human health?. Food Res Int. 2000;33(6):449-59.

[7] Rodriguez-Campos J, Escalona-Buendia $\mathrm{H}$, Orozco-Avila I, Lugo-Cervantes E, JaramilloFlores M. Dynamics of volatile and non-volatile compounds in cocoa (Theobroma cacao L.) during fermentation and drying processes using principal components analysis. Food Res Int. 2011;44(1):250-8.

[8] Schinella G, Mosca S, Cienfuegos-Jovellanos E, Pasamar MA, Muguerza B, Ramon D, et al. Antioxidant properties of polyphenol-rich 
cocoa products industrially processed. Food Res Int. 2010;43(6):1614-23.

[9] Mayorga-Grossa AL, Quirós-Guerrero LM, Fourny G, Vaillanta F. An untargeted metabolomics assessment of cocoa beans during fermentation. Food Res Int. Apr 2016; In press.

[10] Garcia L, Guarda D, Chavez J, Rios R, Chia J. Farmer participatory and on-station selection activities carried out at Universidad Nacional Agraria de la Selva, Peru. In: Eskes AB, editor. Collavorative and participatory approaches to cocoa variety improvement. Final report of the $\mathrm{CFC} / \mathrm{ICCO} /$ Bioversity project on "Cocoa productivity and quality improvement: a participatory approach" (2004-2010). CFC, Amsterdan, The Netherlands/ICCO, London, UK/Bioversity International, Rome, Italy. p. 102-7.

[11] Federación Nacional de Cacaoteros Departamento de Investigación. Protocolo para fermentación de muestras de cacao. May 2012.

[12] Carrión-Santos J. Estudio de factibilidad para la producción y comercialización de cacao (Theobroma cacao L.) variedad CCN-51, Jama-Manabí. Quito, Ecuador: Universidad San Francisco de Quito; 2012.

[13] Maisincho-Asqui MP. Fermentación de cacao (Theobroma cacao) variedad CCN-51 inoculando Acetobacter. Ambato, Ecuador: Universidad Técnica de Ambato; 2006.

[14] Pallares-Pallares A, Perea-Villamil JA, LópezGiraldo LJ. Impacto de las condiciones de beneficio sobre los compuestos precursores de aroma en granos de cacao (Theobroma cacao L) del clon CCN-51. Respuestas. 2016;21(1):120-33.

[15]Pasamar MA, Ibarra A, CienfuegosJovellanos E, Laghi S, inventores/solicitantes. Natraceutical Sa, solicitante. Proceso de obtención de extractos de cacao con elevado contenido de polifenoles. España Patente WO 2007/138118 A1. 2007 Dic 06.

[16] Cadena T, Herrera Y. Evaluación del efecto del procesamiento del cacao sobre el contenido de polifenoles y su actividad antioxidante (Trabajo de pregrado). Bucaramanga: Universidad Industrial de Santander. Facultad de Química; 2008.

[17] Wollgast J. The contents and effects of polyphenols in chocolate. Qualitative and quantitative analyses of polyphenols in chocolate and chocolate raw products as well as evaluation of potential implications of chocolate consumption in human health [Doctoral dissertation]. Gießen: University of Gießen. Faculty of Agricultural and Nutritional Sciences; 2004.

[18] Huang D, Ou B, Hampsch-Wodill M, Flanagan $\mathrm{J}$, Prior R. High-throughput assay of oxygen radical absorbance capacity (ORAC) using a multichannel liquid handling system coupled with a microplate fluorescence reader in 96-well format. J Agric Food Chem. 2002;50(16):443744.

[19] Cienfuegos-Jovellanos E, Quiñones $M$, Muguerza B, Moulay L, Miguel M, Amaya A. Antihypertensive effect of a polyphenolrich cocoa powder industrially processed to preserve the original flavonoids of the cocoa beans. J Agric Food Chem. 2009;57(14):615662.

[20]Ki Won L, Young Jun K, Hyong Joo L, Chang Young L. Cocoa has more phenolic phytochemicals and a higher antioxidant capacity than teas and red wine. J Agric Food Chem. 2003;51(25):7292-5.

[21] Prior R, Wu X, Schaich K. Standardized methods for the determination of antioxidant capacity and phenolics in foods and dietary supplements. J Agric Food Chem. 2005;53(10):4290-302.

[22] Redovnikovic I, Delonga K, Mazor S, Dragovic-Uzelac V, Caric M, Vorkapic-Furac J. Polyphenolic content and composition and antioxidative activity of different cocoa liquors. Czech J Food Sci. 2009;27(5):330-7.

[23] Wollgast J, Pallaroni L, Agazzi ME, Anklam E. Analysis of procyanidins in chocolate by reversed-phase high-performance liquid chromatography with electrospray ionisation mass spectrometric and tandem mass spectrometric detection. J Chromatogr A. 2001;926(1):211-20.

[24]Efraim P, Tucci ML, Pezoa-Garcia NH, Haddad R, Eberlin M. Teores de compostos fenólicos de sementes de cacaueiro de diferentes genótipos. Braz J Food Technol. 2006;9(4):229-36.

[25] Hammerstone J, Lazarus S, Mitchell A, Rucker $\mathrm{R}$, Schmitz $\mathrm{H}$. Identification of procyanidins in cocoa (Theobroma cacao) and chocolate using high-performance liquid chromatography/ mass spectrometry. J Agric Food Chem. 1999;47(2):490-6. 
[26]Nazaruddin R, Seng L, Hassan O, Said M. Effect of pulp preconditioning on the content of polyphenols in coco beans (Theobroma cacao) during fermentation. Ind Crops Prod. 2006;24(1):87-94.

[27] Efraim P, Pezoa-Garcia NH, Jardim DCP, Nishikawa A, Haddad R, Eberlin MN. Influência da fermentação e secagem de amêndoas de cacau no teor de compostos fenólicos e na aceitação sensorial. Ciênc Tecnol Aliment. 2010;30(1):142-50.

[28] Kyi TM, Wan Daud WR, Mohammad AB, Wahid Samsudin M, Hassan Kadhum AA, Meor Talib MZ. The kinetics of polyphenol degradation during the drying of malaysian cocoa beans. Int J Food Sci Technol. 2005;40(3):323-31.

[29] Tomás-Barbean F, Cienfuegos-Jovellanos E, Marn A, Muguerza B, Gil-Izquierdo A, Cerd B, et al. A new process to develop a cocoa powder with higher flavonoid monomer content and enhanced bioavailability in healthy humans. $J$ Agric Food Chem. May 2007; 55(10): 3926-35.

[30] Kim H, Keeney P. (-)-Epicatechin content in fermented and unfermented cocoa beans. J Food Sci. 1984;49(4):1090-2.

[31] Afoakwa E, Quao J, Takrama F, Budu A, Saalia F. Changes in total polyphenols, o-diphenols and anthocyanin concentrations during fermentation of pulp pre-conditioned cocoa (Theobroma cacao) beans. Int Food Res J. 2012;19(3):1071-7.

[32] Serra Bonvehi J, Ventura Coll F. Evaluation of bitterness and astringency of polyphenolic compounds in cocoa powder. Food Chem. 1997;60(3):365-70.

[33] Misnawi JS, Jamilah B, Nazamid S. Oxidation of polyphenols in unfermented and partly fermented cocoa beans by cocoa polyphenol oxidase and tyrosinase. J Sci Food Agric. Apr 2002;82(5):559-66.

[34] Godoy Chivatá JE. Obtención de antioxidantes a partir de grano de cacao: condiciones favorables de inactivación de la enzima polifenol oxidasa, extracción de polifenoles y ajuste de parámetros cinéticos (Trabajo de pregrado). Bucaramanga, Colombia: Universidad Industrial de Santander; 2015.

[35] Gil Quintero JA. Estabilidad y actividad antioxidante de catequinas presentes en cacaos colombianos durante los procesos de pre industrialización (Tesis de Maestría). Medellin: Universidad de Antioquia. Facultad de Química Farmaceutica; 2012.

[36] Adamson G, Lazarus S, Mitchell A, Prior R, Cao G, Jacobs P, et al. HPLC method for the quantification of procyanidins in cocoa and chocolate samples and correlation to total antioxidant capacity. J Agric Food Chem. 999;47(10):4184-8.

[37] Miller K, Stuart D, Smith N, Lee C, McHale $\mathrm{N}$, Flanagan $\mathrm{J}$, et al. Antioxidant activity and polyphenol and procyanidin contents of selected commercially available cocoa-containing and chocolate products in the United States. J Agric Food Chem. 2006;54(11):4062-8.

[38] Counet C, Ouwerx C, Rosoux D, Collin S. Relationship between procyanidin and flavor contents of cocoa liquors from different origins. J Agric Food Chem. 2004;52(20):6243-9.

[39] Ortega N, Romero MP, Macia A, Reguant J, Angles N, Morello JR, et al. Obtention and characterization of phenolic extracts from different cocoa sources. J Agric Food Chem. 2008;56(20):9621-7.

[40] Counet C, Collin S. Effect of the number of flavanol units on the antioxidant activity of procyanidin fractions isolated from chocolate. J Agric Food Chem. 2003;51(23):6816-22.

[41] Schroeter $H$, Heiss C, Balzer J, Kleinbongard P, Keen C, Hollenberg N, et al. (-)-Epicatechin mediates beneficial effects of flavanol-rich cocoa on vascular function in humas. Proc Natl Acad Sci U S A. 2006;103(4):1024-9. 